\title{
FRB as Products of Accretion Disc Funnels
}

\author{
J. I. Katz, ${ }^{1 \star}$ \\ ${ }^{1}$ Department of Physics and McDonnell Center for the Space Sciences, Washington University, St. Louis, Mo. 63130 USA
}

7 April 2018

\begin{abstract}
The repeating FRB 121102, the only FRB with an accurately determined position, is associated with a variable persistent radio source consistent with a low luminosity active galactic nucleus. I suggest that FRB originate in the accretion disc funnels of intermediate mass black holes. Narrowly collimated radiation is emitted along the wandering instantaneous angular momentum axis of accreted matter. We observe this emission as a fast radio burst when it sweeps across the direction to the observer. This model constrains the mass of the black hole to values below those of galactic nuclei. It predicts, in contrast to neutron star (pulsar or SGR) models, that repeating FRB will not be periodic and will be co-located with persistent but variable radio sources resulting from the off-axis emission. The model is analogous, on smaller spatial, lower mass and accretion rate and shorter temporal scales, to AGN making double radio sources, with FRB corresponding to blazars in which the jets point toward us.
\end{abstract}

Key words: radio continuum: general, accretion, accretion discs

\section{INTRODUCTION}

Most FRB models have been based on neutron stars. FRB have been suggested to be higher energy versions of pulsar pulses, powered by neutron star rotational energy, or the result of SGR-like dissipation of magnetostatic energy (see Katz (2016a) for a review). Pulsar models require extreme values of rotation rate and magnetic field to account for FRB peak powers if these are bounded by their spin-down power (see, however, Katz (2017b)). A possible loophole, a wandering collimated beam (Katz 2017a), mitigates this problem but narrow collimation may be inconsistent with a diverging fan of magnetic field lines. The open field lines of a rapidly rotating neutron star diverge widely even near its surface. SGR-based models must be reconciled with the nondetection of a FRB during the giant flare of SGR 1820-06 (Tendulkar, Kaspi \& Patel 2016; Katz 2016b).

This paper proposes an entirely different model based on accretion onto an "intermediate mass" $\left(\sim 10^{2}-10^{6} M_{\odot}\right)$ black hole. This model scales down an AGN rather than scaling up (in energy) a radio pulsar. The model still involves a wandering narrow beam to mitigate the power requirement of isotropic emission. The comparatively large black hole mass permits a high accretion rate and accretional luminosity, although these cannot be estimated without more detailed knowledge of the environment. The narrow funnels of a thick accretion disc (Frank, King \& Raine 2002) confine the relativistic (Katz 2014) radiating particles to a narrow jet, and their radiation to a narrow beam.

* E-mail katz@wuphys.wustl.edu
The assumed narrowly collimated beam is analogous to the jets emitted by AGN, micro-quasars, GRB, SS433 and even protostars. The beam wanders (Katz 2017a) because it is aligned with the disc's instantaneous angular momentum axis that follows the angular momentum of accreting matter. Wandering disc angular momentum is plausible for a intermediate-mass black hole accreting from a chaotic medium such as a giant molecular cloud in a starburst galaxy. This is unlike a supermassive black hole in a galactic nucleus that accretes from a disc aligned with the galactic symmetry plane. When the beam sweeps across the direction to an observer, a burst is observed. The requirement that intrinsic burst durations not exceed a millisecond constrains the mass of the black hole.

\section{IMPLICATIONS OF THE PERSISTENT SOURCE}

The repeating FRB 121102 is associated with a variable persistent radio source (Chatterjee, et al. 2017) and identified with a dwarf galaxy at $z=0.193$ (Tendulkar et al. 2017). The persistent radio source "varies by tens of percent on days timescales" (Chatterjee, et al. 2017); they measured rates of change as high as 7\%/day, implying a characteristic time of 14 days, and suggest it is a low luminosity AGN. The persistent source has a projected separation from the FRB of $<40$ pc (Marcote et al. 2017) in a dwarf galaxy about 100 times larger, indicating co-location in space and a causal connection rather than an accidental association.

Rapid variability argues against pulsar 
wind nebula and supernova remnant models (Murase, Kashiyama \& Mészáros 2016; Kashiyama \& Murase 2017; Marcote et al. 2017; Metzger, Berger \& Margolit 2017; Dai, Wang \& Yu 2017; Beloborodov 2017; Waxman 2017) of the persistent source. Radio emission from the best studied young Galactic pulsar wind nebula, the Crab Nebula, (Aller \& Reynolds 1985) and young supernova remnant, Cas A, (Dent, Aller \& Olsen 1974) decays very gradually, at a rate $\alpha / T$, where $T$ is their age and $\alpha$ is a factor $\mathcal{O}(1)$. If the decay is a power law function of time $\alpha$ is the exponent. There is no evidence of shorter term variability in these objects, nor would that be expected because of the large size of the nebulæ and remnants and the long synchrotron energy loss time of electrons that radiate at radio frequencies. The variability of the persistent source, consisting of a flare above a steadier background, is reminiscent of the behavor of AGN rather than of scintillation, but even if variability is the result of scintillation an AGN remains consistent with the observations.

Even a much younger hypothetical pulsar wind nebula or supernova remnant associated with FRB 121102, whose age is at least four years and size is $\gtrsim 10^{17} \mathrm{~cm}$ (at typical supernova expansion speed $\sim 10^{9} \mathrm{~cm} / \mathrm{s}$ ), would not vary nearly as fast as the persistent counterpart. The time scale of variation could be shorter if the bulk radiating plasma were expanding towards us relativistically, but this is possible only for a pulsar wind nebula unconfined by surrounding matter. Its radius would be $\geq 4$ light-years and free expansion would be inconsistent with any plausible ambient density; there is no evidence of behavior of this kind in Galactic objects.

Rapid variability and the observed (Chatterjee, et al. 2017) nonthermal radio spectrum suggest an accreting black hole, as in an AGN. The source's comparatively low luminosity $\nu L_{\nu} \approx 6 \times 10^{38} \mathrm{ergs} / \mathrm{s}$ at $\nu=3 \mathrm{GHz}$, as well as its presence in a dwarf galaxy of mass estimated to be only $4-7 \times 10^{7} M_{\odot}$, indicate a much lower black hole mass than in AGN. These values may be compared to the luminosity at $2.6 \mathrm{GHz}$ of $3 \mathrm{C} 273 \nu L_{\nu}=7 \times 10^{43} \mathrm{ergs} / \mathrm{s}$ (Soldi et al. 2008) and black hole mass of $\sim 7 \times 10^{9} M_{\odot}$ (Paltani \& Turler 2005) suggesting a giant elliptical host galaxy of mass $\sim 10^{12}-10^{13} M_{\odot}$ (Martel et al. 2003). Rough scaling by a factor $\sim 10^{5}$ suggests the origin of FRB 121102 in accretion onto an intermediate mass black hole.

\section{MAKING FRB}

Accretion, whether on stellar-mass objects or supermassive black holes, is observed to produce fluctuating but not impulsive radiated power. Hence I adopt the assumption of Katz (2017a) that the emission is very narrowly collimated and that the observed bursts result from the sweeping of this narrow beam across the direction to the observer. This assumption was made in order to mitigate the demands on the energetics of a pulsar-like source, but here is applied to a source powered by accretion onto a black hole.

The most accurately measured jets, those of SS433, wander in angle by a few degrees about their mean precessional motion (Katz \& Piran 1982). Because SS433 accretes from its companion in a binary star whose orbital plane is fixed, this indicates that at least some wandering is an intrinsic property of accretion discs. If FRB accrete from dense interstellar or protostellar clouds that are likely to be "turbulent" or "chaotic" in density and velocity in regions of active star formation, the direction of the accreted angular momentum may wander through large angles.

\section{ENERGETICS}

In this model the radiated energy is supplied by accretion. The mean power of bursts depends on their duty factor $D$, which is only known (and even there only very roughly) for the repeating FRB 121102, for which it is $\sim 10^{36} \mathrm{ergs} / \mathrm{s}$ during active periods when bursts are separated by intervals $\sim 1$ minute. This is small compared to the power $\nu L_{\nu} \sim$ $6 \times 10^{38} \mathrm{ergs} / \mathrm{s}$ at $3 \mathrm{GHz}$ of the persistent source. If the persistent power is derived from accretion onto a black hole, the accretion rate must be $\gtrsim 10^{19} \mathrm{~g} / \mathrm{s}$. The inequality results from the omission of unknown luminosity outside the $3 \mathrm{GHz}$ band, the unknown efficiency (Waxman (2017) suggested it may be substantial) of producing radio radiation and from the fact that in super-Eddington flows the accretion rate is unbounded while the emergent luminosity saturates around the Eddington limit as a result of radiation trapping (Katz 1977).

A black hole of mass $M$ moving at supersonic speed $v$ through a homogeneous cloud of density $\rho$ at rest accretes at the rate

$$
\begin{aligned}
\dot{M} & \approx \rho \frac{(G M)^{2}}{v^{3}} \\
& \approx 10^{19}\left(\frac{M}{100 M_{\odot}}\right)^{2}\left(\frac{n_{H}}{10^{5} \mathrm{~cm}^{-3}}\right)\left(\frac{10 \mathrm{~km} / \mathrm{s}}{v}\right)^{3} \mathrm{~g} / \mathrm{s},
\end{aligned}
$$

where $n_{H}$ is the density of hydrogen atoms. The second and third factors in parentheses are plausibly $\mathcal{O}(1)$ for a dense molecular cloud. The first factor in parentheses may be $\gg 1$. This classical result (due to Bondi) is unlikely to be applicable because interstellar gas is surely spatially heterogeneous and may have significant velocity gradients; if it were applicable, then the accreted matter would have no angular momentum about the black hole and would not form a disc (or jet or beam).

The fact that accreting black holes, either of stellar mass or supermassive in AGN, produce jets and often appear to be surrounded by discs, establishes the importance of heterogeneity of the gas distribution and of angular momentum to the accretion process. Unfortunately, this is not understood in detail and no predictive theory exists; for example, the low luminosity of $\mathrm{Sgr} \mathrm{A}^{*}$ at the center of our Galaxy is unexplained. We have almost no information about the environment of FRB 121102 beyond its presence in a dwarf galaxy (for example, is it in a dense molecular cloud?), so it is not possible to say more than that accretion sufficient to power the persistent source is not excluded.

\section{OPENING ANGLES}

The duty factor $D$ of a repeating FRB (for FRB that have not been observed to repeat only an upper bound on $D$, approximately the length of the burst divided by the duration 
of observation, can be set) is related to the opening angle $\theta$ :

$\theta \approx \sqrt{\Omega D}$

where $\Omega$ is the solid angle over which the beam wanders. Optimistically taking $\Omega=4 \pi$ yields $\theta \approx 10^{-2}$ for the repeating FRB 121102 , and $\theta \lesssim 3 \times 10^{-4}$ for the best observed other FRB.

These estimates may be compared to observed jet opening angles (Hughes 1991). In the microquasar/X-ray source Sco X-1 an upper bound of about $1^{\circ}$ may be estimated from the size of the radio lobes (the jet itself is not detected) (Fomalont et al. 1983), while in other microquasars jets may be as narrow as $\sim 2^{\circ}$ (Miller-Jones, Fender \& Nakar 2006). Parsec-scale jets in AGN may have opening angles of $\sim 1^{\circ}$ (Bridle \& Perley 1984; Owen et al. 1989; Bridle et al. 1994; Perlman et al. 1999; Pushkarev, et al. 2009).

These values are larger (much larger in the case of nonrepeating $\mathrm{FRB}$ ) than the beam widths required to explain the observed duty factors. However, the observed opening angles are those of radiation produced by the interaction of the accelerated jet with surrounding matter. This interaction region may be a turbulent layer much wider than the jet itself, broadened as turbulence propagates into the ambient medium. It is likely to be wider than the core of energetic particles or radiation that is not directly observed. Even in SS433, where the observed emission lines are ascribed to the entire (subrelativistic) jet, the radiation may be emitted by ions outside a much narrower jet core that are repeatedly ionized and recombined by interaction with surrounding matter that scatters them from the core.

\section{BOUNDS ON BLACK HOLE MASSES}

The characteristic time scale or duration of a burst is the time required for a beam of width $\theta$ to sweep through its width

$\Delta t \gtrsim \frac{2 G M \theta}{c^{3} \alpha} \sim \frac{2 G M}{c^{3} \alpha \gamma}$,

where $\gamma \sim 1 / \theta$ is the Lorentz factor of the accelerated particles and $\alpha$ is the friction factor (ratio of viscous stress to isotropic pressure) of the disc (Frank, King \& Raine 2002). Here we have multiplied the characteristic inner disc relaxation time $2 G M /\left(c^{3} \alpha\right)$ by $\theta$ to allow for the fact that reorientation by an angle $\theta$, if the disc is supplied by a turbulent flow, is sufficient to begin or end a burst.

Estimating, as before, $\theta \sim \sqrt{4 \pi D}$, Eq. 3 becomes

$\frac{M}{M_{\odot}} \lesssim 100 \frac{\Delta t_{-3} \alpha}{\sqrt{4 \pi D}}$

where $\Delta t_{-3} \equiv \Delta t / 10^{-3}$ s. Typical estimates of $\alpha$ are $\sim 10^{-2}$ (Frank, King \& Raine 2002), but measurements of the rapid "nodding" motion of the jets of SS433 indicate an effective $\alpha \sim 1$ for the propagation of orientational information to the jets (Katz et al. 1982).

Substituting $D \sim 10^{-5}$ for the repeating FRB 121102 and a possible $D \sim 10^{-8}$ for a non-repeating FRB yields bounds on the masses of their black holes $M$

$\frac{M}{M_{\odot}} \lesssim \begin{cases}10^{4} \Delta t_{-3} \alpha & \text { FRB } 121102 \\ 3 \times 10^{5} \Delta t_{-3} \alpha & \text { non-repeaters. }\end{cases}$
The bounds are less strict for smaller values of $D$. By definition, only an upper limit on $D$ can be set for a non-repeater, so that if $D$ is much smaller than the nominal $10^{-8}$ the bound on $M$ for non-repeaters is relaxed.

The bound for FRB 121102 is firmer, although its numerical value depends (not sensitively) on the uncertain evaluation of $D$. Despite this uncertainty, we conclude that in the accretion funnel model the black hole has a mass intermediate between the stellar masses of black holes in binary $\mathrm{X}$-ray sources and the supermassive black holes powering AGN at the centers of galaxies.

\section{DISCUSSION}

The black hole accretion funnel model predicts:

(i) FRB are accompanied by persistent but variable radio sources, their out-of-beam radiation. These persistent sources are analogous to the radiation of AGN whose jets are not pointed toward us (AGN that are not blazars). An example of such an AGN with its jets and radio lobes fortuitously appears near the bottom of Fig. 2a of Chatterjee, et al. (2017).

(ii) Repeating FRB will not be periodic, because (unlike in models based on rotating neutron stars) there is no clock.

(iii) FRB will be associated with very soft X-ray/extreme UV sources produced by accretion discs around intermediate mass black holes. Such radiation is strongly absorbed in the Galactic plane and difficult to observe from FRB 121102 at low Galactic latitude, but may be observable from other FRB.

No model of FRB, or even of classic radio pulsars, has yet satisfactorily explained their coherent emission, so this must be left to future work.

\section{ACKNOWLEDGEMENTS}

I thank J. Eilek for useful discussions.

\section{REFERENCES}

Aller, H. D. \& Reynolds, S. P. 1985 ApJ 293, L73.

Beloborodov, A. M. 2017 ApJ submitted arXiv:1702.08644.

Bridle, A. H. \& Perley, R. A. 1984 ARA\&A 22, 319.

Bridle, A. H. et al. 1994 AJ 108, 766.

Chatterjee, S. et al. 2017 Nature 541, 58 doi:10.1038/nature20797.

Dai, Z. G., Wang, J. S. \& Yu, Y. W. ApJ in press arXiv:1702.05831.

Dent, W. A., Aller, H. D. \& Olsen, E. T. 1974 ApJ 188, L11.

Fomalont, E. B., Geldzahler, B. J., Hjellming, R. M. \& Wade, C. M. 1983 ApJ 275, 802.

Frank, J., King, A. R. \& Raine, D. J. 2002 Accretion Power in Astrophysics, Cambridge U. Press, Cambridge.

Hughes, P., ed. 1991 Beams and Jets in Astrophysics, Cambridge U. Press, Cambridge.

Kashiyama, K. \& Murase, K. 2017 ApJ 839, L3.

Katz, J. I. 1977 ApJ 215, 265.

Katz, J. I. 2014 Phys. Rev. D 89, 103009.

Katz, J. I. 2016a Mod. Phys. Lett. A 31, 1630013.

Katz, J. I. 2016b ApJ 826, 226.

Katz, J. I. 2017a MNRAS doi:10.1093/mnrasl/slx014 arXiv:1611.01243. 
Katz, J. I. 2017b MNRAS doi:10.1093/mnrasl/slx052 arXiv:1702.02161.

Katz, J. I., Anderson, S. F., Margon, B. \& Grandi, S. A. 1982 ApJ 260, 780.

Katz, J. I. \& Piran, T. 1982 Ap. Lett. 23, 11.

Marcote, B. et al. 2017 ApJ 834, L8.

Martel, A. R. et al. 2003 AJ 125, 2964.

Metzger, B. D., Berger, E. \& Margolit, B. 2017 ApJ in press arXiv:1701.02370.

Miller-Jones, J. C. A., Fender, R. P. \& Nakar, E. 2006 MNRAS $367,1432$.

Murase, K., Kashiyama, K. \& Mészáros, P. 2016 MNRAS 461, 1498

Owen, F. N. et al. 1989 ApJ 340, 698.

Paltani, S. \& Turler, M. 2005 A\&A 435, 811.

Perlman, E. S. et al. 1999 AJ 117, 2185.

Pushkarev, A. B., Kovalev, Y. Y., Lister, M. L. \& Savolainen, T. 2009 A\&A 507, L33.

Soldi, S. et al. 2008 A\&A 486, 411.

Tendulkar, S. P. Kaspi, V. M. \& Patel, C. 2016 ApJ 827, 59.

Tendulkar, S. P. et al. 2017 ApJ 834, L7.

Waxman, E. 2017 ApJ submitted arXiv:1703.06723.

This paper has been typeset from a $\mathrm{T}_{\mathrm{E}} \mathrm{X} / \mathrm{LAT}_{\mathrm{E}} \mathrm{X}$ file prepared by the author. 